\title{
Superação de dormência em sementes de Bombax malabaricum D.C. (Malvaceae)
}

FERNANDES-DE-CAMPOS, K.A.'; SAPATINI, J.R. ${ }^{1,}$ PEDROSO-DE-MORAES, C. ${ }^{*}$

${ }^{1}$ Centro Universitário Hermínio Ometto (UNIARARAS). Av. Dr. Maximiliano Baruto, 500, Jd. Universitário, CEP 13607-339, Araras, SP, Brasil. *Autor para correspondência: pedroso@uniararas.br

\begin{abstract}
RESUMO: A espécie Bombax malabaricum, popularmente conhecida por paineira-vermelha, apresenta metabólitos secundários de interesse medicinal. Como outras espécies da família Malvaceae, suas sementes possuem dormência tegumentar, o que dificulta sua propagação sexuada. Assim, o presente trabalho objetivou superar a dormência de sementes de $B$. malabaricum por métodos físicos e químicos. Os métodos utilizados na escarificação mecânica foram: abrasão do tegumento com lixa e corte com tesoura na região oposta ao hilo. Para a escarificação química foram utilizados: ácido clorídrico $(\mathrm{HCl})$, ácido nítrico $\left(\mathrm{HNO}_{3}\right)$, ácido sulfúrico $\left(\mathrm{H}_{2} \mathrm{SO}_{4}\right)$, hidróxido de sódio $(\mathrm{NaOH})$ e hidróxido de potássio $(\mathrm{KOH})$ por um período de 30 minutos. Também houve imersão de sementes em $40 \mathrm{mg} \mathrm{L}^{-1} \mathrm{de} \mathrm{GA}_{3}$ pelo mesmo período. Foram utilizadas quatro repetições por tratamento, constituídas de 25 sementes por placa e conduzidas em Câmara de Germinação. Os dados obtidos foram utilizados para o cálculo da Germinabilidade, Índice de Velocidade de Germinação, Tempo Médio de Germinação, e Porcentagem de Sementes Deterioradas. $\mathrm{O} \mathrm{HNO}_{3}$ mostrou-se como o melhor tratamento na variável Germinabilidade $(83,5 \%)$, seguido do $\mathrm{HCl}(71,5 \%)$ e das técnicas de escarificação mecânica, com lixa $(71,5 \%)$ e tesoura $(67,5 \%)$. O $\mathrm{HNO}_{3}$ também apresentou o menor número de sementes deterioradas (3,5\%); $\mathrm{o} \mathrm{H}_{2} \mathrm{SO}_{4}$ foi o tratamento que mais causou danos às sementes $(36,5 \%)$.
\end{abstract}

Palavras-Chave: Paineira-vermelha, Germinação, Escarificação, Ácido Nítrico.

\begin{abstract}
Dormancy breaking in seeds of Bombax malabaricum D.C. (Malvaceae). The species Bombax malabaricum, popularly known as red-cotton tree, presents secondary metabolites of medicinal interest. Like other species of the Malvaceae, their seeds have tegumentary dormancy, which hinders their sexual propagation. Thus, this study aimed to break the dormancy of $B$. malabaricum through physical and chemical strategies. For mechanical scarification of the seeds it were employed: abrasion of the tegument with sandpaper and cut with scissors in the region opposite to the hilum. For chemical scarification: hydrochloric acid $(\mathrm{HCl})$, nitric acid $\left(\mathrm{HNO}_{3}\right)$, sulphuric acid $\left(\mathrm{H}_{2} \mathrm{SO}_{4}\right)$, sodium hydroxide $(\mathrm{NaOH})$ and potassium hydroxide $(\mathrm{KOH})$ for a period of 30 minutes. The seeds were also subjected to immersion in 40 $\mathrm{mg} \mathrm{L}^{-1}$ of $\mathrm{GA}_{3}$ for the same period. Four replicates of 25 seeds were used for each treatment and then they were conducted for calculation in a B.O.D. germination Chamber. The results obtained were applied in order to calculate: Germination, Germination Speed Index, Average Time of Germination and Percentage of Damaged Seeds. The $\mathrm{HNO}_{3}$ proved to be the best treatment in the Germination trials $(83.5 \%)$, followed by the $\mathrm{HCl}(71.5 \%)$ and the mechanical scarification techniques with sandpaper $(71.5 \%)$ and scissors $(67.5 \%)$. The $\mathrm{HNO}_{3}$ also showed the lowest percentage of damaged seeds (3.5\%); The $\mathrm{H}_{2} \mathrm{SO}_{4}$ was the treatment that caused more damage to the seeds $(36.5 \%)$.
\end{abstract}

Keywords: Red-cotton tree, Germination, Scarification, Nitric acid.

\section{INTRODUÇÃO}

Bombax malabaricum D.C. ou Bombax ceiba Linn. (sinonímia) (Malvaceae) constitui espécie arbórea originária da Índia e Indochina, sendo, comumente conhecida como paineira-vermelha, salmali e paineira-vermelha-da-Índia (Lorenzi et al.,
2003; Mub, 2008). A espécie é amplamente utilizada como ornamental, principalmente na composição de jardins e parques, tanto isoladamente quanto formando maciços (Lorenzi et al., 2003). Caracterizase por ser uma espécie arbórea caducifólia de 15-20 
$\mathrm{m}$ de altura, de tronco espesso e com acúleos agudos aglomerados na base. Suas folhas apresentam-se dispostas espiraladamente, compostas, palmadas com cinco folíolos elípticos de superfície ondulada e de pecíolo vermelho bronzeado; tais órgãos também se apresentam decíduos. As flores são vermelhas e possuidoras de cinco pétalas carnosas de superfície serosa e brilhante, dispostas ao longo de grandes ramos (Lorenzi et al., 2003).

A espécie apresenta metabólitos secundários que promovem uma gama de ações antifúngicas e antibacterianas, apresentando estruturas químicas com diferentes ações quando comparados com substâncias convencionais usadas para controle do crescimento microbiano (Girija et al., 2010). Compostos medicinais são preparados de praticamente todas as partes da planta. As raízes possuem propriedades afrodisíacas e são utilizadas em casos de deficiência seminal (Panda, 2004) e no tratamento de disenterias (Kchare, 2007). As sementes são utilizadas no tratamento de varicela e varíola (Panda, 2004). As folhas, como antiinflamatório e no tratamento de erupções cutâneas, como acne (Kchare, 2007). Frutos secos jovens apresentam propriedades que os permitem ser usados como expectorantes e diuréticos, com uso em casos de calculo renal e inflamações crônicas (Girija et al., 2010) e as flores possuem propriedades adstringentes, sendo usadas na dermatologia e no tratamento de esplenomegalia (Panda, 2004).

A multiplicação da espécie é realizada exclusivamente por sementes as quais apresentam impermeabilidade do tegumento à água, tipo de dormência comumente constatada em sementes da família Malvaceae (Kramer \& Kozlowski, 1972; Popinigis, 1985). Diversos métodos têm sido utilizados na superação desse tipo de dormência, sendo os mais utilizados as escarificações mecânicas e químicas (Popinigis, 1985; Brasil, 2009). A escarificação mecânica consiste no atrito das sementes contra uma superfície abrasiva ou raspagem de uma pequena parte ou corte do tegumento (Carvalho, 2003). Já a química, consiste em submergir as sementes em substâncias ácidas ou alcalinas por um determinado tempo, visando à corrosão deste tecido protetor. Tais métodos visam proporcionar a embebição das sementes para que se dê início ao processo germinativo (Fowler \& Bianchetti, 2000).

Esta pesquisa teve por objetivo determinar o melhor método para a superação de dormência das sementes de B. malabaricum, haja vista que a maioria dos órgãos dessa espécie apresenta importância medicinal, sendoutilizados tradicionalmente para o tratamento de várias patologias e disfunções orgânicas.

\section{MATERIAL E MÉTODOS}

\section{Material botânico}

Para a realização do estudo foram utilizadas 1.100 sementes de Bombax malabaricum, recémcolhidas, oriundas de cinco matrizes presentes no Arboreto do Centro Universitário Hermínio Ometto - Uniararas.

A região de Araras encontra-se sob clima tropical, sazonal, com verão chuvoso e inverno seco, descrito como Cwa na classificação climática de Köppen. As chuvas não ultrapassam 30 mm durante o mês mais seco e a temperatura do mês mais quente oscila entre $19^{\circ} \mathrm{C}$ e $29^{\circ} \mathrm{C}$. Nesta região, há predomínio do Latossolo Vermelho-Amarelo, com manchas mais férteis de Latossolo Vermelho Escuro (Magini \& Chagas, 2003).

\section{Tratamentos}

Os tratamentos utilizados para o ensaio foram:

- escarificação mecânica com lixa n 60 por 1 minuto e desponte com tesoura na região oposta ao hilo;

- escarificação química, por 30 minutos, pela utilização de ácido clorídrico $37 \%$ (P.A.);

- escarificação química, por 30 minutos, pela utilização de ácido nítrico $65 \%$ (P.A.);

- escarificação química, por 30 minutos, pela utilização de ácido sulfúrico $98 \%$ (P.A.);

- escarificação química, por 30 minutos, pela utilização de hidróxido de sódio a $5 \%$;

- escarificação química, por 30 minutos, pela utilização de hidróxido de potássio a $5 \%$;

- tratamento por 30 minutos com $\mathrm{GA}_{3} 40$ $\mathrm{mg} \cdot \mathrm{L}^{-1}$;

- tratamento controle (imersão em água destilada por 30 minutos, em temperatura ambiente).

Posteriormente, um total de 100 sementes / tratamento foram lavadas em água corrente e secas em papel absorvente, sendo distribuídas em quatro grupos de 25 unidades, em quatro placas de Petri, previamente esterilizadas, forradas com duas folhas de papel de filtro nas quais foram adicionados 15 $\mathrm{mL}$ de água destilada, totalizando quatro repetições por tratamento.

As placas foram mantidas em Câmara de Germinação B.O.D. (MA 403), sob temperatura de $25 \pm 2^{\circ} \mathrm{C}$, luz branca fornecida por lâmpadas fluorescentes a $32,85 \mu \mathrm{mol} . \mathrm{m}^{-2} . \mathrm{s}^{-1}$ ao nível das sementes sob foto período de 12 horas. O monitoramento foi diário e sementes com radículas visíveis a olho nu e com curvamento geotrópico positivo foram consideradas germinadas. Os dados obtidos foram utilizados para o cálculo da Germinabilidade (G\%), Índice de Velocidade de 
Germinação (IVG), Tempo Médio de Germinação (TM) (Labouriau \& Agudo, 1987) e Percentagem de Sementes deterioradas (SD\%).

Para a análise estatística dos dados foram submetidos à análise de variância (ANOVA) e as médias comparadas pelo teste de Tukey a $5 \%$ de significância, com o auxílio do aplicativo BioEstat 5.3.

\section{RESULTADOS E DISCUSSÃO}

\section{Germinação e sementes deterioradas}

Entre os tratamentos empregados, os melhores resultados para a variável Germinabilidade (G\%) foram observados utilizando-se $\mathrm{HNO}_{3}$, seguido de escarificação mecânica com lixa, escarificação química em $\mathrm{HCl}$ e escarificação com tesoura (Tabela 1.). Os demais tratamentos, com exceção do $\mathrm{H}_{2} \mathrm{SO}_{4}$, ainda que inferiores aos outros, foram superiores ao tratamento controle (água destilada). $\mathrm{O}$ ácido nítrico, mesmo sendo um ácido forte e corrosivo (Peruzzo \& Canto, 1999), atuou de maneira a promover a germinação de $B$. malabaricum sem que, no entanto, se observassem danos ao embrião, um dos maiores problemas em se tratando de métodos de escarificação química (Nunes et al., 2006).

Em sementes de Urena lobata L. (Malvaceae), também foi observado início de processos germinativos após a utilização de tratamentos prévios com $\mathrm{HNO}_{3}$ (Figueiredo \& Popinigis, 1979). Albrecht (1981), no entanto, observou redução na germinação de sementes de
Mimosa scabrella Benth (Fabaceae) submetidas a tratamento prévio em $\mathrm{HNO}_{3}(70 \%)$ por 30 minutos. O mesmo autor observou coloração avermelhada nas sementes, supondo tratar-se da ação do ácido na queima das substâncias de reserva, o que não foi observado no presente trabalho.

$\mathrm{O}$ tratamento com HCl mostrou-se bastante eficiente na promoção germinativa, com resultados inferiores apenas aos observados no tratamento com ácido nítrico e semelhantes à escarificação mecânica com lixa (Tabela 1), o mesmo observado em sementes de Oxalis hirsutíssima (Mart. \& Zucc) (Oxalidaceae), que apresentaram melhora germinativa considerável quando em contato prévio por 40 segundos com $\mathrm{HCl}$ (Coelho et al., 2000). Postula-se que, em casos de dormência tegumentar, o efeito corrosivo causado por substâncias ácidas seja benéfico, permitindo o inicio do processo de embebição e, baseando-se nos baixos índices de sementes deterioradas (SD\%), é possível inferir que o $\mathrm{HCl}$ atua dessa maneira na espécie testada. Em contrapartida, sementes de Urena lobata L. (Malvaceae) apresentam índices elevados de sementes deterioradas quando submetidas ao tratamento citado (Figueiredo \& Popinigis, 1979) resultados semelhantes aos observados por Dousseau et al. (2007) que notaram efeitos deletérios em sementes de Zehyeria montana Mart. (Bignoniaceae) utilizando-se de $\mathrm{HCl}$, mesmo resultado que observaram no tratamento com $\mathrm{H}_{2} \mathrm{SO}_{4}$. Efeitos negativos, nesses casos, podem ser causados em razão de haver porosidades no tegumento que tendem a acelerar a absorção dos

TABELA 1 - Valores médios obtidos para Germinabilidade (G\%), Índice de Velocidade de Germinação (IVG), Tempo Médio de Germinação (TM) e Porcentagem de Sementes Deterioradas (SD\%) para sementes de Bombax malabaricum após tratamentos. CV $(\%)=$ Coeficiente de Variação.

\begin{tabular}{ccccc}
\hline Tratamentos & $\mathbf{G}(\%)$ & SD (\%) & IVG & TM \\
& & & & \\
$\mathbf{H}_{2} \mathbf{0}$ & $35,5 \pm 0,5 \mathrm{~F}^{1}$ & $24,5 \pm 0,5 \mathrm{C}$ & $1,01 \pm 0,05 \mathrm{G}$ & $07,75 \pm 0,5 \mathrm{C}$ \\
$\mathbf{H C l}$ & $71,5 \pm 0,4 \mathrm{~B}$ & $17,5 \pm 0,4 \mathrm{D}$ & $3,63 \pm 0,04 \mathrm{D}$ & $05,25 \pm 0,5 \mathrm{D}$ \\
$\mathbf{H}_{2} \mathbf{S O}_{4}$ & $06,5 \pm 0,5 \mathrm{G}$ & $36,5 \pm 0,5 \mathrm{~A}$ & $0,73 \pm 0,05 \mathrm{H}$ & $01,25 \pm 0,4 \mathrm{~F}$ \\
$\mathbf{H N O}_{3}$ & $83,5 \pm 0,3 \mathrm{~A}$ & $03,5 \pm 0,5 \mathrm{E}$ & $8,25 \pm 0,01 \mathrm{~A}$ & $04,75 \pm 0,5 \mathrm{D}$ \\
$\mathrm{GA}_{3}$ & $47,5 \pm 0,5 \mathrm{D}$ & $25,5 \pm 0,5 \mathrm{C}$ & $1,16 \pm 0,00 \mathrm{~F}$ & $09,25 \pm 0,5 \mathrm{~B}$ \\
$\mathrm{NaOH}_{\mathbf{K O H}}$ & $43,5 \pm 0,3 \mathrm{E}$ & $27,5 \pm 0,4 \mathrm{~B}$ & $1,17 \pm 0,00 \mathrm{~F}$ & $10,25 \pm 0,5 \mathrm{AB}$ \\
$\mathbf{L i x a}$ & $47,5 \pm 0,5 \mathrm{D}$ & $25,5 \pm 0,5 \mathrm{C}$ & $1,24 \pm 0,00 \mathrm{E}$ & $10,75 \pm 0,4 \mathrm{~A}$ \\
Tesoura & $71,5 \pm 0,5 \mathrm{~B}$ & $02,5 \pm 0,5 \mathrm{E}$ & $6,98 \pm 0,05 \mathrm{~B}$ & $02,75 \pm 0,5 \mathrm{E}$ \\
$\mathbf{C V}(\%)$ & $67,5 \pm 0,4 \mathrm{C}$ & $02,5 \pm 0,5 \mathrm{E}$ & $5,25 \pm 0,50 \mathrm{C}$ & $02,75 \pm 0,5 \mathrm{E}$ \\
\hline
\end{tabular}

${ }^{1}$ Números seguidos por letras iguais na mesma coluna, não diferem entre si pelo teste de Tukey a $5 \%$ de significância. 
ácidos e causar danos ao embrião (Pedroso-deMoraes et al., 2012).

Em B. malabaricum, o ácido sulfúrico $\left(\mathrm{H}_{2} \mathrm{SO}_{4}\right)$ mostrou-se como o tratamento com menor taxa germinativa e maior número de sementes deterioradas (Tabela 1); no entanto, em diversas espécies, o uso desse ácido na escarificação apresenta resultados satisfatórios, como o acréscimo germinativo em sementes de Malva aegypta L. (Malvaceae) observado por Figueiredo \& Popinigs (1979) utilizando-se de $\mathrm{H}_{2} \mathrm{SO}_{4}$ a $96 \%$ por 30 minutos, em sementes de Senna macranthera DC. (Ex collad.) H.S. Irwin \& Barn (Fabaceae) (Eschiapatti-Ferreira \& Perez, 1977) e em sementes de Sterculia striata St. Hil (Malvaceae) submetidas à escarificação ácida por $\mathbf{3 0}$ minutos (Mata et al., 2010). Os resultados mostram que, devido às particularidades germinativas de cada espécie, considerando os diversos tipos de dormência (Cardoso, 2009) e as características de cada tegumento, resultados variáveis em relação aos mesmos tratamentos químicos em diferentes espécies vegetais são observados.

Reguladores vegetais com ação giberélica são comumente utilizados para superação de dormência morfofisiológica em sementes (Fernandes et al., 2012). Tal dormência se caracteriza pela presença de um embrião subdesenvolvido, e de um processo fisiológico a ser estimulado ou inibido para o início do fenômeno germinativo (Cardoso, 2009). Determinadas espécies, como Lavandula augustifolia Mill. (Lamiaceae) apresentam, após embebição em $\mathrm{GA}_{3}$ (200 mg L-1), índices germinativos muito superiores aos observados pelo controle, provavelmente em resposta ao efeito de indução enzimática que ocasiona a degradação de reservas endospermáticas, etapa necessária ao início do processo germinativo e que pode ser estimulada pela aplicação exógena de reguladores de ação giberélica (Kerbauy, 2004). Quando em contato com $\circ \mathrm{GA}_{3}$ as sementes de B. malabaricum, no entanto, apresentaram resposta intermediária em relação aos demais tratamentos, ainda que com resultados superiores ao controle (Tabela 1 ), o que leva a suposição que maiores concentrações deste regulador, possivelmente possam ser empregados para a superação de dormência em sementes da espécie. O mesmo se aplica aos tratamentos químicos $\mathrm{KOH}$ e $\mathrm{NaOH}$, que apresentaram para espécie testada, resultados intermediários, ainda que superiores ao controle. Acredita-se que o uso de bases cause permeabilização do tegumento devido à possível corrosão causada, o que pode favorecer a promoção da germinação (Albrecht, 1981). Alguns autores recomendam ainda o uso de soluções básicas, defendendo a hipótese de que, mesmo embriões desenvolvidos, aptos a germinar, não o fazem em razão do inadequado grau de acidez (Toledo \& Marcos-Filho, 1977). Gurgel-Filho (1947) observou acréscimo germinativo em sementes de Delonix regia (Bojer ex Hook.) Raf. Rafinesque, C.S. (Fabaceae) utilizando-se de tratamentos prévios compostos por $\mathrm{NaOH}$ a $4 \%$ por 24 horas, o mesmo que Abrecht (1981) em sementes de $M$. scabrella, com tratamento prévio a $\mathrm{NaOH}$ a $2 \%$.

Os tratamentos de escarificação mecânica apresentaram acréscimos significativos no percentual de germinação e números reduzidos de sementes deterioradas em relação ao controle (Tabela 1), o que também foi observado por Johnston (1971) utilizando-se de tratamentos de escarificação envolvendo cortes no tegumento em sementes de espécies da família Malvaceae. Dousseau et al. (2007) observaram resultados semelhantes em sementes de Z. montana submetidas a escarificação manual antecedida por lavagem em água corrente, acréscimos também observados por Castro et al. (1996) em sementes de Brachiaria decubens Stapf. (Poaceae). No mesmo trabalho (Castro et al., 1996), foram observados decréscimos na germinação correlacionados ao aumento da intensidade do processo de escarificação, resultado semelhante ao descrito em sementes de Stylosanthes guianensis SW (Poaceae). Dessa maneira, a intensidade e a metodologia propostas no presente trabalho, baseando-se nos resultados obtidos, mostramse eficientes na promoção da germinação de B. malabaricum. Como nos tratamentos de escarificação mecânica, resultados semelhantes foram obtidos em relação ao número de sementes deterioradas no uso de $\mathrm{HNO}_{3}$ (Tabela 1); esses se apresentam como os tratamentos que causaram menores danos ao embrião, mostrando-se menos agressivos nas concentrações e períodos de exposição empregados.

Ainda em relação à Percentagem de Sementes Deterioradas (SD\%), o uso de $\mathrm{H}_{2} \mathrm{SO}_{4}$, como dito anteriormente, seguido de $\mathrm{NaOH}$ apresentaram as maiores médias, mostrando serem esses tratamentos menos indicados à espécie testada (Tabela 1). O fato de não terem sido observadas diferenças estatísticas entre os tratamentos controle, $\mathrm{GA}_{3}$ e $\mathrm{KOH}$ leva a postular que os danos causados, nesses casos, devam-se mais ao período de exposição e à ineficácia do tratamento no estímulo à germinação do que à ação corrosiva desses compostos.

\section{Velocidade e Uniformidade de Germinação}

Em se tratando das medidas de velocidade e uniformidade temporal de germinação, $\mathrm{O}^{\mathrm{HNO}_{3}}$ apresentou resultados superiores aos demais no Índice de Velocidade de Germinação (IVG), seguido da escarificação mecânica com lixa, (Tabela 
1). $\mathrm{O}$ tratamento com $\mathrm{H}_{2} \mathrm{SO}_{4}$ e o grupo controle, respectivamente, foram aqueles que apresentaram menor velocidade de germinação. Utilizado como ferramenta para avaliar o vigor de sementes, o IVG se mostra muito útil na interpretação da germinação de um lote de sementes, uma vez que amostras com a mesma porcentagem total de germinação podem apresentar valores diferentes nesse índice, o que indica variações nos padrões de comportamento temporal do lote analisado (Santana \& Ranal, 2004). Já o TM, é uma medida que leva em consideração o número variável de sementes que germinou em cada tempo, o que não é tido em conta pela média aritmética e pode gerar erros de interpretação quanto à uniformidade da germinação (Labouriau, 1983).

Os maiores valores na variável TM foram obtidos pelos tratamentos com $\mathrm{KOH}, \mathrm{NaOH}$, e $\mathrm{GA}_{3}$, As escarificações mecânicas e $\mathrm{O}_{2} \mathrm{SO}_{4}$ apresentaram as médias mais baixas de germinação em relação a essa variável. O hidróxido de potássio forma soluções altamente alcalinas em solventes polares e, como o hidróxido de sódio, fornece ânions altamente nucleofílicos $\left(\mathrm{OH}^{-}\right)$, capazes de, em determinadas situações, destruir ligações polares em materiais orgânicos (Peruzzo \& Canto, 1999), o que pode ter contribuído de alguma forma na uniformidade da germinação observada nos tratamentos (Tabela 1.).

O comportamento das sementes de B. malabaricum em relação aos tratamentos apresentados demonstra a necessidade de emprego de um tratamento prévio para se obter porcentagens razoáveis de germinação, o que leva à constatação e confirmação da ocorrência de dormência na espécie. Os métodos de escarificação mecânica (tesoura e lixa) apresentaram resultados significativos, ainda que inferiores ou semelhantes aos tratamentos ácidos $\left(\mathrm{HNO}_{3}\right.$ e $\left.\mathrm{HCl}\right)$ o que leva a crer não se tratar apenas de dormência física, imposta por envoltórios impermeáveis (Cardoso, 2009). Sabese que, na maioria dos casos, observam-se combinações de diferentes tipos de dormência, o que dificulta a classificação exata. Mais do que a simples ausência de sinais externos germinativos, a dormência é considerada uma modalidade de desenvolvimento, passível de controle metabólico e genômico (Labouriau, 1983); logo, a superação da dormência deve estar relacionada a uma sequência de fatores que ocorrem concomitantemente e que deve ser considerada na metodologia aplicada para cada caso. Levando-se em conta os processos ecológicos, metabólicos e genômicos envolvidos na dormência, diferentes métodos representam pontos favoráveis na classificação do tipo de dormência, na escolha do método de superação ou até mesmo na diferenciação de processos naturais pouco estudados que ocorrem em sementes quiescentes.

Os resultados permitem inferir que o ácido nítrico, pouco usado com a finalidade de superar dormência em sementes, pode proporcionar interessantes resultados, e que, nesse sentido, diferentes combinações de tempos de exposição e concentrações do ácido devem ser consideradas em experimentos para a espécie.

Conclui-se que o uso de ácido nítrico apresentou-se como o melhor tratamento para superar a dormência da espécie Bombax malabaricum, apresentando maior taxa germinativa, velocidade de germinação e menor índice de sementes deterioradas, em relação aos demais tratamentos realizados. $\mathrm{O} \mathrm{H}_{2} \mathrm{SO}_{4}$ mostrou-se, altamente deletério às sementes de $B$. malabaricum.

\section{REFERÊNCIAS}

ALBRECHT, J.M.F. Estudos sobre a germinação de Mimosa scabrella Benth. ("Bracatinga") e Acacia mearnsii DeWild ("Acácia-negra") em função de tratamentos pré-germinativos. 1981. 76p. Dissertação (Mestrado em Engenharia Florestal) - Departamento de Ciências Agrárias, Universidade Federal do Paraná, Curitiba.

BRASIL, MINISTÉRIO DA AGRICULTURA E REFORMA AGRÁRIA. Regras para análise de sementes. Brasília: SNAD/DNDV/CLAV, 2009. 627p.

CARDOSO, V.J.M. Conceito e classificação da dormência em sementes. Oecologia Brasiliensis, v.13, n.4, p. 619-631, 2009.

CARVALHO, P.E.R. Espécies arbóreas brasileiras. Brasília: Embrapa Informações Tecnológicas, 2003.1039p.

CASTRO, C.R.T. et al. Superação da dormência tegumentar em sementes de Brachiaria decubens Stapf. Revista Ceres, v. 43, n.245, p. 65-75, 1996.

COELHO, M.F.B. et al. Germinação de sementes de azedinha (Oxalis Hirtutissima), espécie medicinal de Mato Grosso do Sul, Brasil. Acta Amazônica, v.30, n.1, p. 3-8, 2000.

DOUSSEAU, S. et al. Superação de dormência em sementes de Zeyheria montana Mart. Ciência e Agrotecnologia, v. 31, n.6, p. 1744-1748, 2007.

ESCHIAPATTI-FERREIRA, M.S.; PEREZ, S.C.J.G.A. Tratamentos para superar a dormência de sementes de Senna macranthera (Collad.) Irwin et Barn. (FabaceaeCaesalpinoideae). Revista Brasileira de Sementes, v.19, n.2, p.231-237, 1977.

FERNANDES, M.R. et al. Morfobiometria carpo seminal e germinação de Lafoensia pacari A. St. Hil. (Lythraceae) exposta a diferentes concentrações de $\mathrm{GA}_{3}$. Semina: Ciências Agrárias, v. 33, suplemento 1, p. 2571-2584, 2012.

FIGUEIREDO, F.J.C.; POPINIGIS, F. Superação da dormência das sementes de malva. Revista Brasileira de Sementes. v.1, n.3, p. 1-14, 1979.

FOWLER, J.A.P.; BIANCHETTI, A. Dormência em sementes florestais. Colombo: Embrapa Florestas, 
2000. 27p.

GIRIJA, K. et al. Evaluation of antimicrobial activity of various bark extracts of Bombax malabaricum. International Journal of Research in Pharmaceutical Sciences, v.1, n.3, p.199-204. 2010.

GURGEL-FILHO, O.A. O faveiro: ensaio sobre a germinação e transplante. Boletim do Serviço Florestal, v.34, n.2, p. 31-51, 1947.

JONHSTON, A. The germination of malvaceous seeds. Tropical Agriculture, v. 26, n.1/6, p. 63-65, 1971.

KCHARE, C.P. Indian medicinal plants. Bagalore: Springer Science/Business Média, 2007. 97p.

KERBAUY, G.B. Fisiologia Vegetal. Rio de Janeiro: Guanabara Koogan, 2004. 452 p.

KRAMER, P.J.; KOZLOWSKI, T.T. Fisiologia das árvores. Lisboa: Fundação Calouste Gulbenkan, 1972. 745p.

LABOURIAU, L.G. A germinação de sementes. Brasília: Organização dos Estados Americanos. Programa Regional de Desenvolvimento Científico e Tecnológico. Série Biologia, 1983. 174p.

LABOURIAU, L.G.; AGUDO, M. On the physiology of germination in seeds in Salvia hispanica L. temperature effects. Anais Academia Brasileira de Ciências, v. 59, n.1, p. 37-56, 1987.

LORENZI, H. et al. Árvores Exóticas no Brasil: madeiras, ornamentais e aromáticas. Nova Odessa: Plantarum, 2003. 384p.

MAGINI, C.; CHAGAS, R.L. Microzoneamento e Diagnóstico Físico-Químico do Ribeirão das Araras,
Araras - SP. São Paulo. Geociências, v. 22, n.2, p. 195-208, 2003.

MATA, M. F. et al. Superação da dormência de sementes de chichá (Sterculia striata A. St. Hil. \& Naudin.) Malvaceae - Sterculioideae. Revista Homem, Espaço e Tempo UEVA. v.1, n.4, p. 214-232, 2010.

MUB, U. A brief introduction to Semal (Bombax ceiba Linn). Goettingen: Rajendra K.C. Dendrology and Wood Science Department. 2008. 14p.

NUNES, Y.R.F. et al. Germinação de sementes de Guazuma ulmifolia Lam. (Malvaceae) e Heteropterys byrsonimifolia A. Juss (Malpighiaceae) sob diferentes tratamentos de escarificação tegumentar. Unimontes Científica, v. 8, n.1, p. 1-18, 2006.

PANDA, H. Handbook on Medicinal Herbs with Uses. Delhi: Asia Pacific Business Press, 2004. 564p.

PEDROSO-DE-MORAES, C. et al. Efeitos da escarificação química e da concentração de nitrogênio sobre a germinação e o desenvolvimento in vitro de Vanilla planifolia Jack ex Andr. (Orchidaceae: Vanilloideae). Acta Botanica Brasílica, v. 26, n.3, p. 714-719, 2012.

PERUZZO, T.M.; CANTO, E.L. Química. São Paulo: Moderna, 1999. 351p.

POPINIGIS, F. Fisiologia da semente. Brasília: AGIPLAN, 1985. 289p.

SANTANA, D.G.; RANAL, M.A. Análise da Germinação: um enfoque estatístico. Brasília: UNB, 2004. 247p.

TOLEDO, F.F.; MARCOS-FILHO, J. Manual das sementes. São Paulo: Agronômica Ceres. 1977. 224p. 